\title{
Association between Tumor Vasculogenic Mimicry and the Poor Prognosis of Gastric Cancer in China: An Updated Systematic Review and Meta-Analysis
}

\author{
Qiujun Guo, ${ }^{1,2}$ Yuan Yuan, ${ }^{1,2}$ Zhichao Jin, ${ }^{1}$ Tao Xu, ${ }^{1}$ Yebo Gao, ${ }^{1,2}$ Huamin Wei, \\ Conghuang Li, ${ }^{1}$ Wei Hou, ${ }^{1}$ and Baojin Hua ${ }^{1}$ \\ ${ }^{1}$ Department of Oncology, Guang'anmen Hospital, China Academy of Chinese Medicine Sciences, No. 5 Beixiange, \\ Xicheng District, Beijing 100053, China \\ ${ }^{2}$ Beijing University of Chinese Medicine, No. 11 North Third Ring Road East, Chaoyang District, Beijing 100029, China \\ Correspondence should be addressed to Conghuang Li; liconghuang@163.com and Baojin Hua; dr.huabaojin@hotmail.com
}

Received 17 June 2016; Accepted 3 August 2016

Academic Editor: Wan-Liang Lu

Copyright ( 2016 Qiujun Guo et al. This is an open access article distributed under the Creative Commons Attribution License, which permits unrestricted use, distribution, and reproduction in any medium, provided the original work is properly cited.

Background. Vasculogenic mimicry can promote tumor growth and metastasis. This article is aimed at conducting a systematic meta-analysis to explore the clinicopathological and prognostic significance of vasculogenic mimicry and gastric cancer. Methods. We searched Pubmed, EMBASE, Cochrane Library, China National Knowledge Infrastructure, and the VIP and Wanfang Database for eligible studies. We manually searched for printed journals and relevant textbooks. Subgroups analyses were performed based on the region, manuscript quality, methods of vasculogenic mimicry identification, pathology, and number of patients. Results. Nine studies with 997 patients were included in this meta-analysis. A significant association was observed between vasculogenic mimicry-positive patients and those with gastric cancer with poor overall survival (hazard ratio $=2.24,95 \%$ confidence interval: 1.45-3.47), poor pathological grading, high tumor node metastasis clinical stage, lymph node metastasis, deep tumor invasion, and distant metastasis. Conclusions. Vasculogenic mimicry is associated with a poor prognosis in patients with gastric cancer in China. Clinical studies with large samples are needed worldwide and standardized protocols should be adopted in the future to achieve a better understanding of the relationship between gastric cancer and vasculogenic mimicry.

\section{Introduction}

Gastric cancer (GC) is a leading cause of death worldwide, accounting for 0.7 million deaths and nearly 1 million newly diagnosed cases in 2012 [1,2]. There is a high incidence of and high mortality from GC in China, making up more than one-third of the world population [3]. Although a variety of treatments such as surgery, chemotherapy, radiation therapy, and targeted therapy have an effect on GC, the 5-year survival rate still remains low, especially in cases of recurrence and metastasis [4].

Vasculogenic mimicry (VM) was first found in melanoma in 1999 , and it indicated that tumor cells can directly generate vascular channels that facilitate tumor perfusion independent of tumor angiogenesis by vascular endothelial cells [5]. Based on the aforementioned features, VM can be distinguished by using the immunohistochemical or histochemical double staining, as VM is recognized as periodic acid-Schiff (PAS) positive and CD31 or CD34 (endothelial markers) negative, whereas classic blood vessels are double positive for PAS and endothelial markers.

VM can promote tumor growth and metastasis, and it is closely related to tumor neovascularization and cancer stem-like cells, which are considered hallmarks of cancer, and they are associated with tumor invasion and drug resistance [6]. Recent studies have shown that VM is associated with a poor prognosis in human tumors [7-11]. A meta-analysis [12] indicated no significant relationship between VM and GC. It only included two eligible studies on GC with 257 patients and minimal valuable clinical evidence. However, 
a recent study indicated that VM may play an important role in the evolution of GC [13]. Based on the aforementioned controversy, we conducted this meta-analysis to evaluate the relationship between VM and the prognosis and clinicopathological features of patients with GC. This study was conducted according to the PRISMA guidelines (see S6 file in Supplementary Material available online at http://dx.doi.org/10.1155/2016/2408645) [14, 15].

\section{Materials and Methods}

2.1. Identification of Eligible Studies. We searched Pubmed, EMBASE, Cochrane Library, China National Knowledge Infrastructure, and the VIP and Wanfang Database for eligible studies without using language limits. The search period was from January 1999 to October 2015. The search terms were as follows: "vasculogenic mimicry or tumour celllined vessels" and "gastric or stomach". We used both Medical Subject Heading terms and free-text words to increase the sensitivity of the search.

In addition to electronic databases, printed journals and relevant textbooks were manually searched in the libraries of Beijing University of Chinese Medicine, Peking Union Medical College, and Guang'anmen Hospital. Specialized experts in particular fields were consulted for necessary supplements as well.

Inclusion criteria were as follows: (1) studies on patients with a histological diagnosis of GC; (2) articles on those with VM-positive primary tumor tissues assessed by the immunohistochemical or histochemical double staining method; and (3) studies aiming to assess the relationship between VM and at least one of the following outcome variables and clinicopathological features: overall survival (OS) time, tumor node metastasis clinical stage, lymph node metastasis, poor pathological grade, blood metastasis, and depth of tumor invasion.

Exclusion criteria were as follows: (1) reviews and single case reports; (2) studies referring to VM but not to humans with VM with gastric cancer; and (3) studies that lacked outcome variables and clinicopathological features.

2.2. Data Extraction and Management. Two independent reviewers (Yebo Gao and Zhichao Jin) extracted the data by using a standardized collection form according to the aforementioned inclusion and exclusion criteria of eligible studies. We recorded the details of eligible studies, including the first author, patients' region, publication year, pathological type, VM assay methods, total cases, clinicopathological features, and outcomes. If there were discrepancies between the two reviewers, a final consensus was reached after discussion with the other author (Yuan Yuan). The hazard ratio (HR) was calculated from the Kaplan-Meier survival curve and 5-year survival outcome events as reported by Tierney et al. [16].

2.3. Methodological Assessment. The methodological assessment of eligible studies was conducted by using the quality scale for biological prognostic factors (S5 file), which was reported previously [17] by two specialists (Huamin Wei and Tao $\mathrm{Xu}$ ) who are experienced in clinical and basic experiments. Disagreements were discussed with another specialist (Conghuang Li).

2.4. Statistical Analyses. Statistical analyses were performed using Review Manager (RevMan) 5.3.5 software (Cochrane Community, London, United Kingdom) and STATA 14 software (STATA Corp., College Station, TX). Dichotomous data of the clinicopathological features were pooled using odds ratios (ORs) with 95\% confidence intervals (CIs). HRs were pooled as inverse variance data with $95 \%$ CI. $P<0.05$ was considered to indicate a statistically significant difference. An observed HR or OR > 1 implied a worse prognosis for the group with VM positivity, and it was considered statistically significant if the values of $95 \%$ confidence intervals did not overlap the value " 1 ." The heterogeneity of the included studies was evaluated by the $\chi^{2}$ and $I^{2}$ tests, and $P<0.10$ or $I^{2}>50 \%$ was defined as heterogeneous. The fixed-effect model was used for merging the homogeneous data, and the random-effects model was suitable for merging the heterogeneous data as previously reported [26]. Publication bias was evaluated by Egger's test (STATA 14) with $P<0.05$ indicating potential bias. The sensitivity analysis was evaluated by reanalyzing the data using different statistical approaches.

\section{Results}

3.1. Characteristics of the Included Studies. Three hundred thirty-five studies, including 37 additional records identified through other sources such as postgraduate dissertations and conference articles, were initially found by using the aforementioned search strategy. One hundred ten duplicate studies were removed, 171 were excluded because they did not study VM, 20 were excluded because they did not study human cancer, and 4 were excluded because they did not study GC. After reading the full text, 21 studies were excluded because they did not mention the relationship between VM and the prognosis or clinicopathology of patients with GC. Finally, 9 studies with 997 patients were included (Figure 1 and Table 1).

Six articles studied the HR between the OS and VM. HRs were calculated using the method previously reported by Tierney et al. [16] (S4 file Table 1). The methodological assessment of eligible studies was conducted as described in Table 2.

\subsection{Results of the Meta-Analysis}

3.2.1. VM Positivity Indicated a Poor Prognosis in Patients with GC. Six studies reported the OS of patients with GC with VM positivity for 715 patients. A significant association was observed between VM positivity and OS. This result suggested that VM positivity may represent a poor prognostic factor for patients with GC (random-effect model: $\mathrm{HR}=2.24$, 95\% CI: 1.45-3.47) (Figure 2). An insignificant heterogeneity was detected among the studies $\left(\mathrm{chi}^{2}=16.94, \mathrm{df}=5, P=\right.$ 0.005 , and $\left.I^{2}=70 \%\right)$. The sensitive analysis was performed by using the fixed-effects model (HR $=2.25$, 95\% CI: $1.82-$ 2.79) (S1 file Figure 1.5). The results of the two models were comparable. 


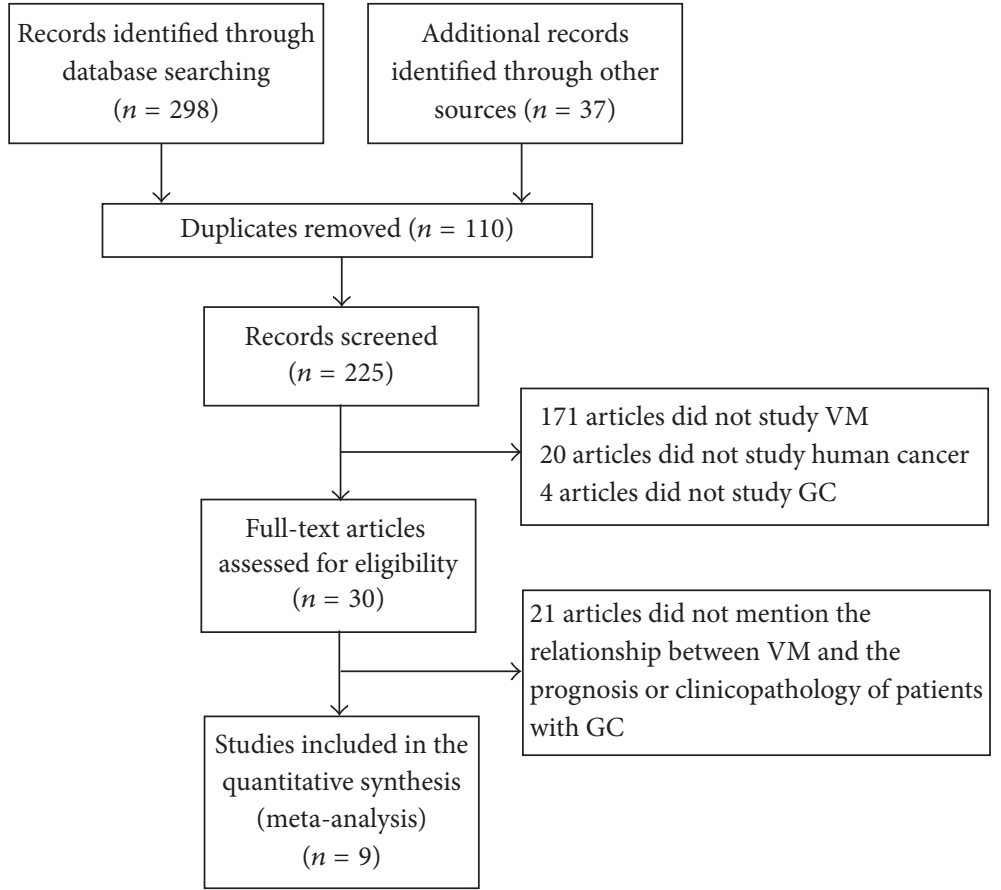

FIGURE 1: Flow diagram of the literature search process. VM, vasculogenic mimicry; GC, gastric cancer.

3.3. Subgroup Analyses. Due to the presence of heterogeneity, subgroups analyses were performed based on the region, methods of VM identification, pathology, and number of patients (Table 3 and S1 file Figures 1.1-1.5).

We detected a significant association between VM positivity and the OS of patients with GC in inland regions (HR $=2.90,95 \% \mathrm{CI}: 2.23-3.78$ ) but not in coastal regions (HR $=1.38$, 95\% CI: 0.96-1.99). VM positivity was significantly associated with the OS of patients with adenocarcinoma (HR $=2.36,95 \%$ CI: $1.44-3.86$ ) but was not significantly associated with the OS of patients with sarcoma $(\mathrm{HR}=1.67,95 \% \mathrm{CI}$ : $0.72-3.87$ ). The association between VM positivity and the OS of patients was present in studies with more than 100 or fewer than 100 subjects $(\mathrm{HR}=1.96,95 \% \mathrm{CI}$ : 104-3.66; $\mathrm{HR}=2.85,95 \% \mathrm{CI}: 1.74-4.67)$. In addition, analysis of the subgroups using different VM detection methods showed a poor OS in the PAS-positive and CD34-negative staining subgroup (HR 2.60, 95\% CI: 1.61-4.19) and PAS-positive and CD31-negative staining subgroup (HR 2.10, 95\% CI: 1.074.10). Significant heterogeneity existed among the studies with the methods of VM identification, pathological type, and sample size subgroups; however, there was no significant heterogeneity in the subgroups for the study region.

Furthermore, results of the sensitivity analyses showed that changing the study effect model did not change the results of the pooled analyses of OS.

3.4. Associations between VM Positivity and the Clinicopathological Characteristics of Patients with GC. The prognostic significance of VM positivity in the TNM clinical stage was evaluated in 6 studies $[13,18-20,22,24]$ with 789 patients. The results showed that VM positivity can lead to a high TNM clinical stage (III or IV clinical stage) in patients with GC (random-effects model: $\mathrm{OR}=3.12,95 \% \mathrm{CI}$ : $1.52-6.42$ ) with a significant heterogeneity. In the analysis of 7 studies $[13,18-$ 20, 22-24] with 910 patients, VM positivity was significantly associated with lymph node metastasis in patients with GC (fixed-effect model: $\mathrm{OR}=2.82$, 95\% CI: 2.04-3.92). We evaluated the relationship between VM positivity and the pathological differentiation in 7 studies [13, 18-20, 22-24] with 910 patients. The results showed that VM positivity can induce poorer pathological differentiation (fixed-effect model: $\mathrm{OR}=3.64,95 \% \mathrm{CI}: 2.53-5.24)$. The prognostic significance of VM positivity in patients with blood metastasis was analyzed in 3 studies [19, 22, 24] with 331 patients. We observed a significant relationship between VM positivity and blood metastasis in patients with GC (fixed-effect model: $\mathrm{OR}=4.34,95 \% \mathrm{CI}: 1.57-11.96)$. The depth of invasion in patients with GC tended to correlate with VM positivity in the evaluation of 2 studies [13, 22] with 335 patients (fixedeffect model: $\mathrm{OR}=2.95,95 \% \mathrm{CI}: 1.63-5.35)$. There was no significant heterogeneity between studies on lymph node metastasis, pathological differentiation, blood metastasis, and the depth of invasion. The aforementioned results are presented in Table 4 (Figures S2.1-2.5 and S4 Table 1).

3.5. Publication Bias. The publication bias was assessed by Egger's test, and the results demonstrated no obvious publication bias in our meta-analysis (Figures S3.1-3.5).

\section{Discussion}

VM is found in many kinds of tumors, and it is regarded as a poor prognostic marker in sarcomas [27], melanomas 


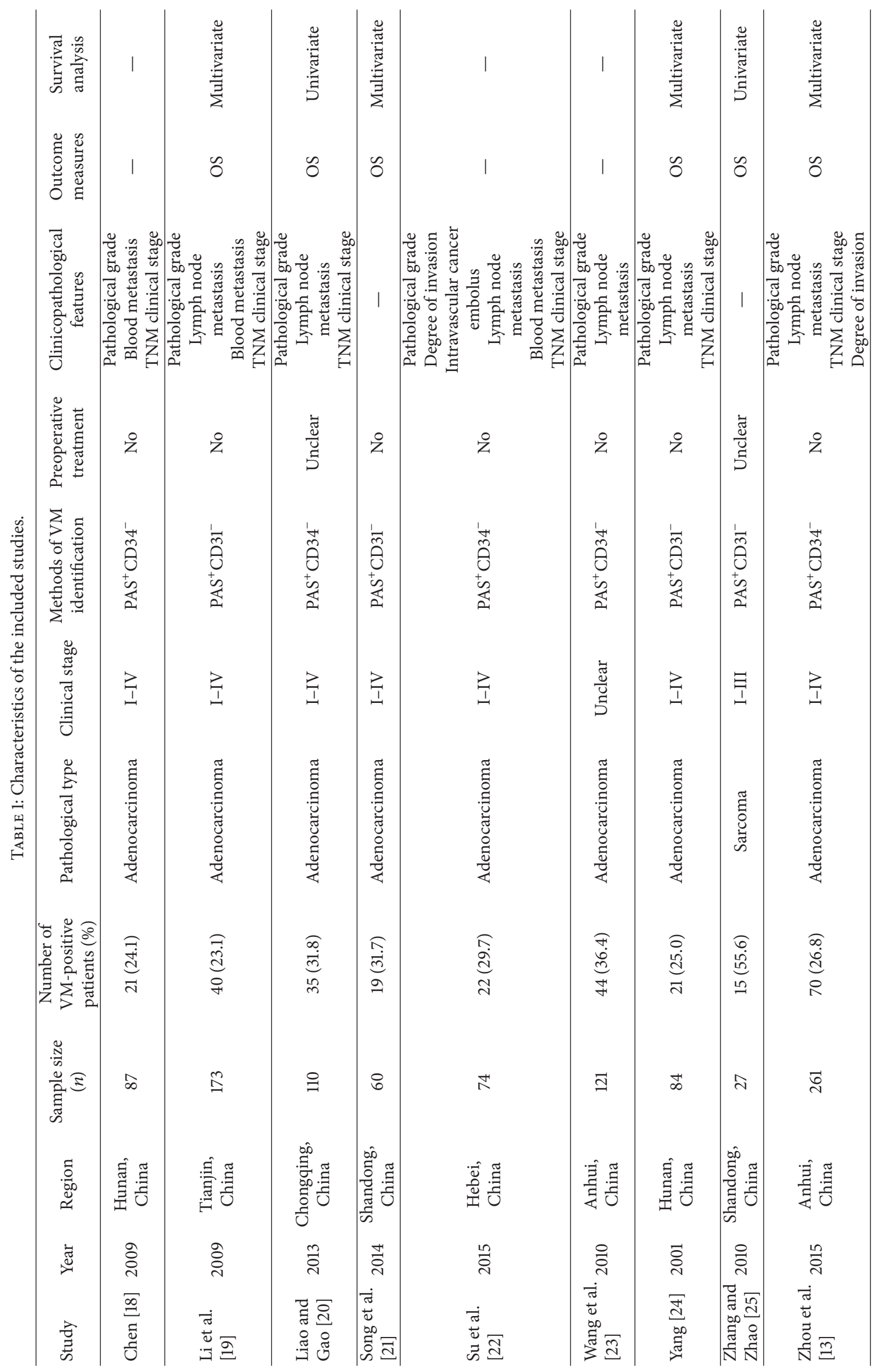


TABLE 2: Quality assessment of the included studies.

\begin{tabular}{|c|c|c|c|c|c|}
\hline Study & Scientific design & Laboratory methodology & Generalizability & Results analysis & Global score $(\%)$ \\
\hline Chen [18] & 9 & 12 & 8 & 0 & 73 \\
\hline Li et al. [19] & 9 & 8 & 10 & 6 & 83 \\
\hline Liao and Gao [20] & 9 & 5 & 5 & 4 & 58 \\
\hline Song et al. [21] & 9 & 8 & 7 & 5 & 73 \\
\hline Su et al. [22] & 9 & 7 & 8 & 0 & 60 \\
\hline Wang et al. [23] & 7 & 5 & 8 & 0 & 50 \\
\hline Yang [24] & 9 & 10 & 8 & 6 & 83 \\
\hline Zhang and Zhao [25] & 8 & 4 & 3 & 5 & 50 \\
\hline Zhou et al. [13] & 9 & 6 & 8 & 5 & 70 \\
\hline
\end{tabular}

\begin{tabular}{|c|c|c|c|c|c|c|c|c|c|c|c|c|}
\hline Study or subgroup & $\begin{array}{c}\text { Log } \\
\text { [hazard ratio] }\end{array}$ & SE & $\begin{array}{l}\text { Experimental } \\
\text { Total }\end{array}$ & $\begin{array}{c}\text { Control } \\
\text { Total }\end{array}$ & Weight & $\begin{array}{c}\text { Hazard ratio } \\
\text { IV, random, } 95 \% \text { CI }\end{array}$ & & & $\begin{array}{l}\text { Iazar } \\
\text { indo }\end{array}$ & $\begin{array}{l}\text { d ratio } \\
\mathrm{m}, 95 \% \mathrm{CI}\end{array}$ & & \\
\hline Li et al., 2009 & 0.1398 & 0.2249 & 40 & 133 & $20.4 \%$ & $1.15[0.74,1.79]$ & & & 二 & $=$ & & \\
\hline Liao and Gao, 2013 & 0.6729 & 0.2546 & 35 & 75 & $19.3 \%$ & $1.96[1.19,3.23]$ & & & & & & \\
\hline Song et al., 2014 & 1.0613 & 0.5415 & 19 & 41 & $10.2 \%$ & $2.89[1.00,8.35]$ & & & & & & \\
\hline Yang, 2001 & 1.4609 & 0.3816 & 21 & 63 & $14.7 \%$ & $4.31[2.04,9.10]$ & & & & & & \\
\hline Zhang and Zhao, 2010 & 0.5128 & 0.4293 & 15 & 12 & $13.2 \%$ & $1.67[0.72,3.87]$ & & & & & & \\
\hline Zhou et al., 2015 & 1.1663 & 0.1745 & 70 & 191 & $22.3 \%$ & $3.21[2.28,4.52]$ & & & & & & \\
\hline Total (95\% CI) & & & 200 & 515 & $100.0 \%$ & $2.24[1.45,3.47]$ & & & & & & \\
\hline \multirow{2}{*}{\multicolumn{7}{|c|}{$\begin{array}{l}\text { Heterogeneity: } \tau^{2}=0.19 ; \chi^{2}=16.94 ; \mathrm{df}=5(P=0.005) ; I^{2}=70 \% \\
\text { Test for overall effect: } Z=3.63(P=0.0003)\end{array}$}} & 0.1 & 0.5 & 1 & 2 & 5 & 10 \\
\hline & & & & & & & \multicolumn{3}{|c|}{ Favours (experime } & \multicolumn{3}{|c|}{ Favours (control) } \\
\hline
\end{tabular}

FIGURE 2: Forest plot of hazard ratios (HRs) in the random-effect model. The HR of overall survival of vasculogenic mimicry- (VM-) positive cancer patients was compared with VM-negative cancer patients. Each individual study is represented by the red square, and the pooled datasets are indicated by the diamond, representing the $95 \%$ confidence interval (CI) of each study. An HR > 1 implied a worse survival for the cancer patients. The size of each study represents the weighting factor (1/standard error [SE]) assigned to it.

[28], hepatocellular carcinomas [29], laryngeal squamous cell carcinomas [30], colorectal cancers [31], gallbladder carcinomas [32], non-small cell lung cancers [33], and osteosarcomas [34]. Although the molecular mechanisms of VM are not fully understood, a study indicated that VM formation was associated with cancer stem cells that improved transendothelial migration in VM-forming cells [35]. Besides, VE-cadherin overexpression and metalloproteinases (MMPs), via their cleavage of laminin, promote adherence of the VM channel wall to tumor cells [36, 37]. Multiple signaling pathways contribute to VM formation. It was shown that vascular endothelial growth factor- (VEGF-) a-EphA2-MMPs were the main pathway for VM formation, and VEGF-a appeared to play an important role in the formation of VM [38]. The VEGF and phosphoinositide 3kinase/AKT pathway also exert positive feedback regulation in the process of VM formation [39]. EphA2 is a protein tyrosine kinase receptor commonly expressed in epithelial cells [40], and it contributes to VM formation by mediating the EphA2/FAK/Paxillin pathway[41-44]. In the Wnt/ $\beta$ catenin pathway, hypoxic conditions and microRNAs also take part in VM formation [45-47].

A previous study with insufficient clinical research indicated that VM was not closely associated with the prognosis of patients with GC [12]. However, our meta-analysis showed that VM was significantly associated with the short OS, poor pathological grading, high TNM clinical stage, lymph node metastasis, deep tumor invasion, and distant metastasis of patients with GC.

The heterogeneity of the included studies drew our attention. To determine the studies with heterogeneity, we applied the random-effects model and fixed-effect model and performed sensitivity analysis. The subgroup analysis showed significant heterogeneity for the methods of VM identification subgroups and those with sample size more than 100 subgroup and adenocarcinoma pathological type subgroup, indicating that the following factors may account for the heterogeneity. Firstly, the quality of the included studies varied, as some studies may be comparatively not well designed. Secondly, VM was detected in different ways (e.g., PAS-positive and CD31- or CD34-negative staining). Differences in the quality of the antibodies, laboratory reagents, conditions, and pathological evaluation standards may have resulted in this heterogeneity. Furthermore, according to our subgroup analysis, we found that a significant association between VM positivity and the OS was detected for gastric adenocarcinoma patients in inland regions in China.

There were some limitations in our study. First, all studies included in our evaluation were conducted in China; hence, the conclusion can only be carefully applied to China or East Asia, not worldwide. Second, there was some heterogeneity among the eligible studies, and their global scores 
TABLE 3: Results of the subgroup analysis of the included studies.

\begin{tabular}{|c|c|c|c|c|c|c|c|c|}
\hline \multirow{2}{*}{ Study subgroups } & \multirow{2}{*}{$\begin{array}{l}\text { Number of } \\
\text { studies }\end{array}$} & \multirow{2}{*}{$\begin{array}{c}\text { Number of } \\
\text { patients }\end{array}$} & \multicolumn{4}{|c|}{ Pooled HR [95\% CI] } & \multicolumn{2}{|c|}{ Heterogeneity } \\
\hline & & & Fixed & $P$ value & Random & $P$ value & $I^{2}(\%)$ & $P$ value \\
\hline \multicolumn{9}{|l|}{ Study region } \\
\hline Coastal region & 3 & 260 & $1.38[0.96,1.99]$ & 0.09 & $1.48[0.92,2.39]$ & 0.11 & 26 & 0.26 \\
\hline Inland & 3 & 455 & $2.90[2.23,3.78]$ & $<0.00001$ & $2.89[1.94,4.30]$ & $<0.00001$ & 47 & 0.15 \\
\hline \multicolumn{9}{|c|}{ Methods of VM identification } \\
\hline $\mathrm{PAS}^{+} \mathrm{CD} 34^{-}$ & 2 & 371 & $2.74[2.07,3.64]$ & $<0.00001$ & $2.60[1.61,4.19]$ & $<0.0001$ & 61 & 0.11 \\
\hline $\mathrm{PAS}^{+} \mathrm{CD} 31^{-}$ & 4 & 344 & $1.72[1.24,2.39]$ & 0.001 & $2.10[1.07,4.10]$ & 0.03 & 70 & 0.02 \\
\hline \multicolumn{9}{|l|}{ Pathological type } \\
\hline Adenocarcinoma & 5 & 688 & $2.30[1.84,2.87]$ & $<0.00001$ & $2.36[1.44,3.86]$ & 0.0007 & 76 & 0.002 \\
\hline Sarcoma & 1 & 27 & $1.67[0.72,3.87]$ & 0.03 & $1.67[0.72,3.87]$ & 0.23 & Not ap & plicable \\
\hline \multicolumn{9}{|l|}{ Sample size $(n)$} \\
\hline$>100$ & 3 & 544 & $2.13[1.68,2.70]$ & $<0.00001$ & $1.96[1.04,3.66]$ & 0.04 & 85 & 0.001 \\
\hline$<100$ & 3 & 171 & $2.85[1.74,4.67]$ & $<0.0001$ & $2.81[1.57,5.05]$ & 0.0005 & 27 & 0.26 \\
\hline
\end{tabular}

VM, vasculogenic mimicry; OR, odds ratio; CI, confidence interval; HR, hazard ratio; PAS, periodic acid-Schiff.

TABLE 4: Meta-analysis of VM and the clinical and pathological features of patients with GC.

\begin{tabular}{|c|c|c|c|c|c|c|c|c|}
\hline \multirow{2}{*}{$\begin{array}{l}\text { Clinical and pathological } \\
\text { features }\end{array}$} & \multirow{2}{*}{$\begin{array}{l}\text { Number of } \\
\text { studies }\end{array}$} & \multirow{2}{*}{$\begin{array}{l}\text { Number of } \\
\text { patients }\end{array}$} & \multicolumn{4}{|c|}{ Pooled OR [95\% CI] } & \multicolumn{2}{|c|}{ Heterogeneity } \\
\hline & & & Fixed & $P$ value & Random & $P$ value & $I^{2}(\%)$ & $P$ value \\
\hline III/IV clinical stage & $\begin{array}{c}6[13,18- \\
20,22,24]\end{array}$ & 789 & $3.35[2.31,4.86]$ & $<0.00001$ & $3.12[1.52,6.42]$ & $<0.0001$ & 65 & 0.01 \\
\hline Lymph node metastasis & $\begin{array}{c}7[13,18- \\
20,22-24]\end{array}$ & 910 & $2.82[2.04,3.92]$ & $<0.00001$ & $2.84[1.95,4.14]$ & $<0.00001$ & 20 & 0.28 \\
\hline Poor differentiation & $\begin{array}{c}7[13,18- \\
20,22-24]\end{array}$ & 910 & $3.64[2.53,5.24]$ & $<0.00001$ & $3.92[2.33,6.59]$ & $<0.00001$ & 35 & 0.16 \\
\hline Blood metastasis & $3[19,22,24]$ & 331 & $3.79[2.14,6.71]$ & $<0.00001$ & $4.34[1.57,11.96]$ & 0.005 & 54 & 0.11 \\
\hline $\mathrm{T} 3 / 4$ invasion & $2[13,22]$ & 335 & $2.95[1.63,5.35]$ & 0.0003 & $3.06[1.29,7.27]$ & 0.01 & 17 & 0.27 \\
\hline
\end{tabular}

$\mathrm{VM}$, vasculogenic mimicry; GC, gastric cancer; OR, odds ratio; CI, confidence interval.

were diverse. These limitations may have influenced our evaluation, but the same results were reached in the sensitivity analysis via different testing models.

In conclusion, VM was associated with the poor prognosis of patients with GC in China, especially for those gastric adenocarcinoma patients in inland regions; however, drugs targeting VM should be studied and used in GC treatment. Furthermore, clinical studies with large samples are needed to evaluate the relationship between GC and VM worldwide, and standardized protocols should be adopted in future studies.

\section{Disclosure}

The funders had no role in the study design, data collection and analysis, decision to publish, or preparation of the manuscript.

\section{Competing Interests}

The authors declare that they have no competing interests.

\section{Authors' Contributions}

Qiujun Guo, Yuan Yuan, and Zhichao Jin contributed equally to this work.

\section{Acknowledgments}

This work was supported by the National Twelfth Five-Year Plan for Science and Technology Support Program of China (no. 2014BAI10B01) and National Natural Science Foundation of China (nos. 81202656, 81273718, and 81403346).

\section{References}

[1] B. W. Stewart and C. P. Wild, “World cancer report 2014," World Health Organization, 2015, http://apps.who.int/bookorders/anglais/detart1.jsp? codlan $=1 \& \operatorname{cod} \mathrm{col}=80 \& \operatorname{cod} \mathrm{cch}=275$.

[2] Z. X. Zheng, R. S. Zheng, S. W. Zhang, and W. Q. Chen, "An analysis of incidence and mortality of stomach cancer in China, 2010," China Cancer, vol. 23, no. 10, pp. 795-800, 2010. 
[3] W. Chen, R. Zheng, P. D. Baade et al., "Cancer statistics in China, 2015," CA: A Cancer Journal for Clinicians, vol. 66, no. 2, pp. 115$132,2016$.

[4] J.-H. Lee, H.-I. Kim, M. G. Kim, T. K. Ha, M.-S. Jung, and S. J. Kwon, "Recurrence of gastric cancer in patients who are disease-free for more than 5 years after primary resection," Surgery, vol. 159, no. 4, pp. 1090-1098, 2016.

[5] A. J. Maniotis, R. Folberg, A. Hesset et al., "Vascular channel formation by human melanoma cells in vivo and in vitro: vasculogenic mimicry," American Journal of Pathology, vol. 155, no. 3, pp. 739-752, 1999.

[6] D. Hanahan and R. A. Weinberg, "Hallmarks of cancer: the next generation," Cell, vol. 144, no. 5, pp. 646-674, 2011.

[7] Z. Yang, B. Sun, X. Zhao et al., "Erythropoietin and erythropoietin receptor in hepatocellular carcinoma: correlation with vasculogenic mimicry and poor prognosis," International Journal of Clinical \& Experimental Pathology, vol. 8, no. 4, pp. 4033-4043, 2015.

[8] C. I. Schnegg, M. H. Yang, S. K. Ghosh, and M.-Y. Hsu, "Induction of vasculogenic mimicry overrides VEGF-A silencing and enriches stem-like cancer cells in melanoma," Cancer Research, vol. 75, no. 8, pp. 1682-1690, 2015.

[9] T. Liu, B. Sun, X. Zhao et al., "OCT4 expression and vasculogenic mimicry formation positively correlate with poor prognosis in human breast cancer," International Journal of Molecular Sciences, vol. 15, no. 11, pp. 19634-19649, 2014.

[10] K. Ren, N. Yao, G. Wang et al., "Vasculogenic mimicry: a new prognostic sign of human osteosarcoma," Human Pathology, vol. 45, no. 10, pp. 2120-2129, 2014.

[11] Y. Zhang, M. M. Baocun Sun, M. B. Xiulan Zhaoet et al., "Clinical significances and prognostic value of cancer stemlike cells markers and vasculogenic mimicry in renal cell carcinoma," Journal of Surgical Oncology, vol. 108, no. 6, pp. 414419, 2013.

[12] Z. Cao, M. Bao, L. Miele, F. H. Sarkar, Z. Wang, and Q. Zhou, "Tumour vasculogenic mimicry is associated with poor prognosis of human cancer patients: a systemic review and meta-analysis," European Journal of Cancer, vol. 49, no. 18, pp. 3914-3923, 2013.

[13] L. Zhou, L. Yu, Z.-Z. Feng et al., "Aberrant expression of markers of cancer stem cells in gastric adenocarcinoma and their relationship to vasculogenic mimicry," Asian Pacific Journal of Cancer Prevention, vol. 16, no. 10, pp. 4177-4183, 2015.

[14] D. Moher, A. Liberati, J. Tetzlaff, and D. G. Altman, "Preferred reporting items for systematic reviews and meta-analyses: the PRISMA statement," PLoS Medicine, vol. 6, no. 7, Article ID e1000097, 2009.

[15] A. Liberati, D. G. Altman, J. Tetzlaff et al., "The PRISMA statement for reporting systematic reviews and meta-analyses of studies that evaluate healthcare interventions: explanation and elaboration," The British Medical Journal, vol. 339, article b2700, 2009.

[16] J. F. Tierney, L. A. Stewart, D. Ghersi, S. Burdett, and M. R. Sydes, "Practical methods for incorporating summary time-toevent data into meta-analysis," Trials, vol. 8, no. 1, article 16, 2007.

[17] E. Steels, M. Paesmans, T. Berghmans et al., "Role of p53 as a prognostic factor for survival in lung cancer: a systematic review of the literature with a meta-analysis," European Respiratory Journal, vol. 18, no. 4, pp. 705-719, 2001.
[18] H. J. Chen, "Primary research of Vasculogenic Mimicry in gastric cancer tissue," Database: China Dissertation Database, 2009 (Chinese), http://d.wanfangdata.com.cn/Thesis/J018372.

[19] M. Li, Y. Gu, Z. Zhanget et al., "Vasculogenic mimicry: a new prognostic sign of gastric adenocarcinoma," Pathology \& Oncology Research, vol. 16, no. 2, pp. 259-266, 2009.

[20] S. Liao and Q. Gao, "Expressions and clinical significance of vasculogenic mimicry and related protein Mig-7 and MMP-2 in gastric carcinoma," Chinese Journal of Cellular and Molecular Immunology, vol. 29, no. 2, pp. 194-196, 2013.

[21] Y.-Y. Song, L.-D. Sun, M.-L. Liu et al., "STAT3, p-STAT3 and HIF-1 $\alpha$ are associated with vasculogenic mimicry and impact on survival in gastric adenocarcinoma," Oncology Letters, vol. 8, no. 1, pp. 431-437, 2014.

[22] H. L. Su, H. H. Su, P. X. Zhang, Q. Lin, K. Chen, and F. L. Liu, "The expression of c-Met and p-Akt in gastric cancer and their correlations with VM," The Practical Journal of Cancer, vol. 30, no. 12, pp. 1771-1774, 2015 (Chinese).

[23] X. Q. Wang, S. W. Zhang, X. Wang, and Q. Wu, "Pilot study of vasculogenic mimicry in gastric adenocarcinoma," Acta Universitatis Medicinalis Anhui, vol. 5, pp. 709-711, 2010 (Chinese).

[24] Z. Yang, A preliminary study of vasculogenic mimicry in gastric cancer [M.S. thesis], Nanhua University, Chiayi, Taiwan, Database: China Dissertation Database, 2001, http://d.wanfangdata.com.cn/Thesis/D521263.

[25] Y. S. Zhang and H. Zhao, "Tumor vasculogenic mimicry in gastric sarcoma tissues," China Foreign Fedical Trestment, vol. 30, no. 90, 2010 (Chinese).

[26] J. Y. Zhou, C. Xin, Y. P. Mouet et al., "Robotic versus laparoscopic distal pancreatectomy: a meta-analysis of short-term outcomes," PLoS ONE, vol. 11, no. 3, Article ID e0151189, 2016.

[27] B. Sun, S. Zhang, X. Zhao, W. Zhang, and X. Hao, "Vasculogenic mimicry is associated with poor survival in patients with mesothelial sarcomas and alveolar rhabdomyosarcomas," International Journal of Oncology, vol. 25, no. 6, pp. 1609-1614, 2004.

[28] A. Van Beurden, R. F. Schmitz, C. M. van Dijk, and C. I. Baeten, "Periodic acid Schiff loops and blood lakes associated with metastasis in cutaneous melanoma," Melanoma Research, vol. 22, no. 6, pp. 424-429, 2012.

[29] Z. Yang, B. Sun, X. Zhao et al., "Erythropoietin and erythropoietin receptor in hepatocellular carcinoma: correlation with vasculogenic mimicry and poor prognosis," International Journal of Clinical and Experimental Pathology, vol. 8, no. 4, pp. 4033-4043, 2015.

[30] P. Lin, W. Wang, B. C. Sunet et al., "Vasculogenic mimicry is a key prognostic factor for laryngeal squamous cell carcinoma: a new pattern of blood supply," Chinese Medical Journal, vol. 125, no. 19, pp. 3445-3449, 2012.

[31] Z. Liu, B. Sun, L. Qi, H. Li, J. Gao, and X. Leng, "Zinc finger E-box binding homeobox 1 promotes vasculogenic mimicry in colorectal cancer through induction of epithelialto-mesenchymal transition," Cancer Science, vol. 103, no. 4, pp. 813-820, 2012.

[32] W. Sun, Z.-Y. Shen, H. Zhang et al., "Overexpression of HIF-1 $\alpha$ in primary gallbladder carcinoma and its relation to vasculogenic mimicry and unfavourable prognosis," Oncology Reports, vol. 27, no. 6, pp. 1990-2002, 2012.

[33] S. Wu, L. Yu, D. Wang et al., "Aberrant expression of CD133 in non-small cell lung cancer and its relationship to vasculogenic mimicry," BMC Cancer, vol. 12, article 535, 2012. 
[34] K. Ren, N. Yao, G. Wang et al., "Vasculogenic mimicry: a new prognostic sign of human osteosarcoma," Human Pathology, vol. 45, no. 10, pp. 2120-2129, 2014.

[35] T. Liu, B. Sun, X. Zhao et al., "USP $44^{+}$cancer stem cell subclones contribute to breast cancer aggressiveness by promoting vasculogenic mimicry," Molecular Cancer Therapeutics, vol. 14, no. 9, pp. 2121-2131, 2015.

[36] A. R. Hess, E. A. Seftor, R. E. B. Seftor, and M. J. C. Hendrix, "Phosphoinositide 3-kinase regulates membrane Type 1matrix metalloproteinase (MMP) and MMP-2 activity during melanoma cell vasculogenic mimicry," Cancer Research, vol. 63, no. 16, pp. 4757-4762, 2003.

[37] M. J. C. Hendrix, E. A. Seftor, P. S. Meltzer et al., "Expression and functional significance of VE-cadherin in aggressive human melanoma cells: role in vasculogenic mimicry," Proceedings of the National Academy of Sciences of the United States of America, vol. 98, no. 14, pp. 8018-8023, 2001.

[38] J. Wang, T. Sun, X. L. Zhao et al., "Functional significance of VEGF-a in human ovarian carcinoma: role in vasculogenic mimicry," Cancer Biology \& Therapy, vol. 7, no. 5, pp. 758-766, 2008.

[39] L. Qin, Y. Ren, A.-M. Chen et al., "Peroxisome proliferatoractivated receptor $\gamma$ ligands inhibit VEGF-mediated vasculogenic mimicry of prostate cancer through the AKT signaling pathway," Molecular Medicine Reports, vol. 10, no. 1, pp. 276-282, 2014.

[40] A. R. Hess, E. A. Seftor, L. M. G. Gardner et al., "Molecular regulation of tumor cell vasculogenic mimicry by tyrosine phosphorylation: role of epithelial cell kinase (Eck/EphA2)," Cancer Research, vol. 61, no. 8, pp. 3250-3255, 2001.

[41] W. Wang, P. Lin, B. Sun et al., "Epithelial-mesenchymal transition regulated by EphA2 contributes to vasculogenic mimicry formation of head and neck squamous cell carcinoma," BioMed Research International, vol. 2014, Article ID 803914, 10 pages, 2014.

[42] X.-S. Lu, W. Sun, C.-Y. Ge, W.-Z. Zhang, and Y.-Z. Fan, "Contribution of the PI3K/MMPs/Ln-5 $\gamma 2$ and EphA2/FAK/Paxillin signaling pathways to tumor growth and vasculogenic mimicry of gallbladder carcinomas," International Journal of Oncology, vol. 42, no. 6, pp. 2103-2115, 2013.

[43] M. Zang, Y. Zhang, B. Zhang et al., "CEACAM6 promotes tumor angiogenesis and vasculogenic mimicry in gastric cancer via FAK signaling," Biochimica et Biophysica Acta-Molecular Basis of Disease, vol. 1852, no. 5, pp. 1020-1028, 2015.

[44] H. Wang, W. Sun, W.-Z. Zhang et al., "Inhibition of tumor vasculogenic mimicry and prolongation of host survival in highly aggressive gallbladder cancers by norcantharidin via blocking the ephrin type a receptor $2 /$ focal adhesion kinase/paxillin signaling pathway," PLoS ONE, vol. 9, no. 5, article e96982, 2014.

[45] X. L. Bai, Q. Zhang, L. Y. Ye et al., "Myocyte enhancer factor 2C regulation of hepatocellular carcinoma via vascular endothelial growth factor and Wnt/ $\beta$-catenin signaling," Oncogene, vol. 34, no. 31, pp. 4089-4097, 2015.

[46] X.-G. Mao, X.-Y. Xue, L. Wang et al., "CDH5 is specifically activated in glioblastoma stemlike cells and contributes to vasculogenic mimicry induced by hypoxia," Neuro-Oncology, vol. 15, no. 7, pp. 865-879, 2013.

[47] H.-Y. Wan, Q.-Q. Li, Y. Zhang et al., "MiR-124 represses vasculogenic mimicry and cell motility by targeting amotL1 in cervical cancer cells," Cancer Letters, vol. 355, no. 1, pp. 148-158, 2014. 


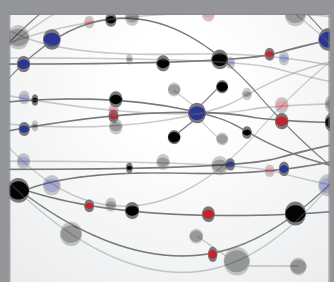

The Scientific World Journal
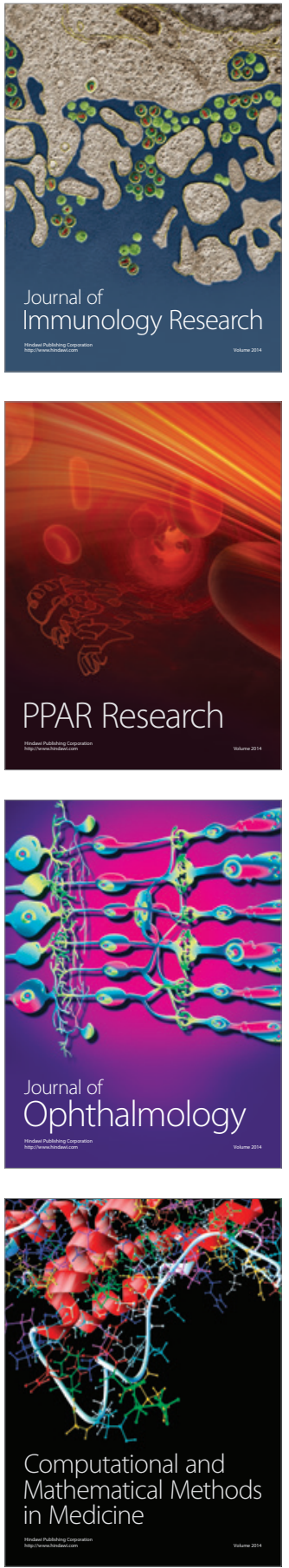

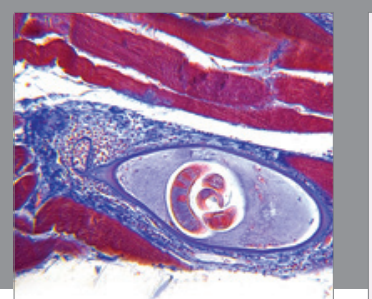

Gastroenterology Research and Practice

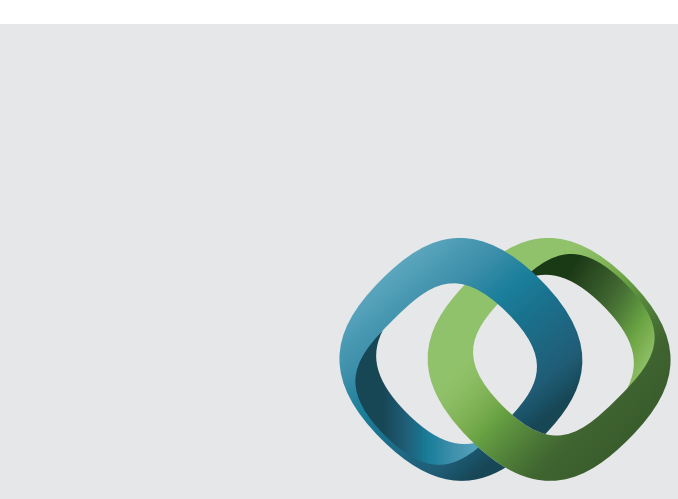

\section{Hindawi}

Submit your manuscripts at

http://www.hindawi.com
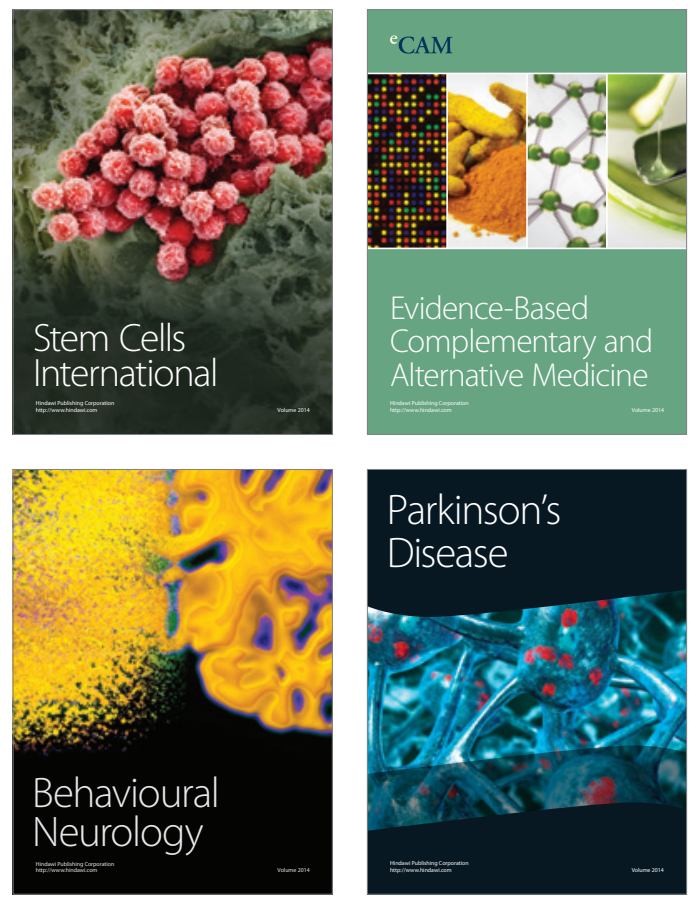
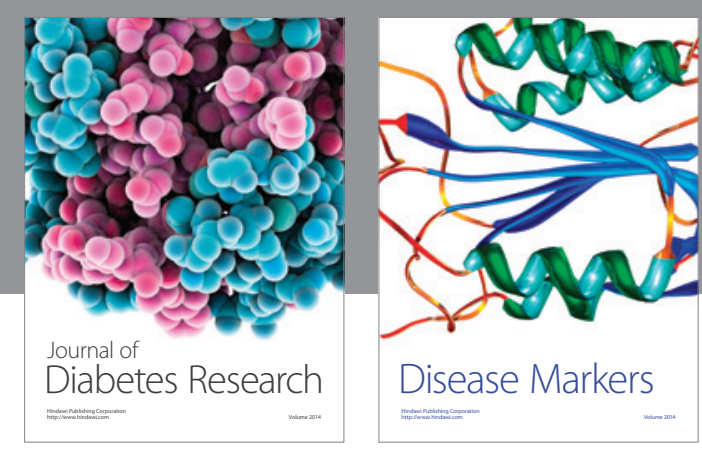

Disease Markers
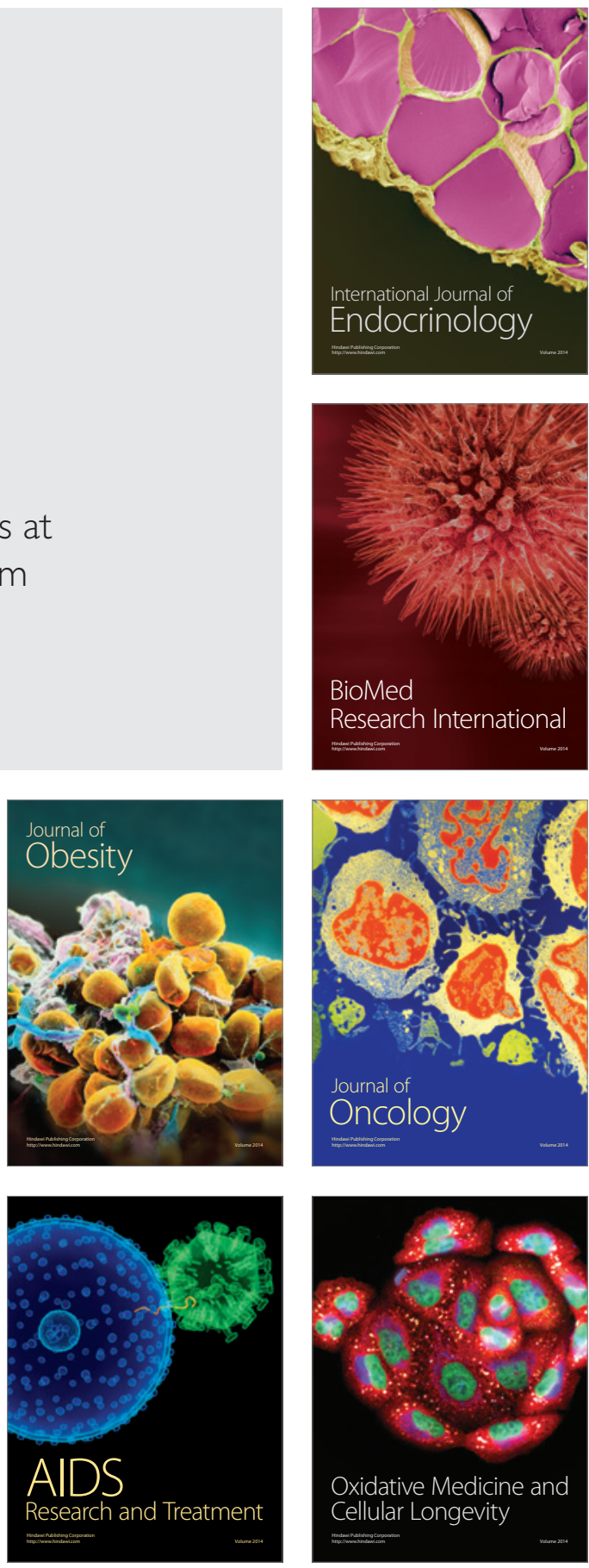